\title{
Effectiveness of Chest CT in Children: CT Findings in Relation to the Clinical Question
}

\section{Wertigkeit der Thorax-CT bei Kindern: Befunde in Abhängigkeit der klinischen Fragestellung}

Authors

Michael EsserD, Ilias Tsiflikas, Mareen Sarah Kraus@, Sabine Hess, Sergios Gatidis, Jürgen F. Schaefer

Affiliation

Diagnostic and Interventional Radiology, Universitätsklinikum

Tübingen, Tübingen, Germany

Key words

thorax, CT-spiral, pediatrics

received 12.04 .2021

accepted 27.07.2021

published online 14.10 .2021

Bibliography

Fortschr Röntgenstr 2022; 194: 281-290

DOI 10.1055/a-1586-3023

ISSN 1438-9029

(C) 2021. Thieme. All rights reserved.

Georg Thieme Verlag KG, Rüdigerstraße 14,

70469 Stuttgart, Germany

Correspondence

Dr. Michael Esser

Radiology, Eberhard-Karls-Universität Tübingen, Hoppe-Seyler-Straße 3, 72074 Tübingen, Germany

Tel.: +49/70 71/2986677

michael.esser@med.uni-tuebingen.de

\section{ZUSAMMENFASSUNG}

Ziel Abschätzung der klinischen Wertigkeit der Thorax-CT bei Kindern auf Grundlage der Verdachtsdiagnose und positiven, negativen und inkonklusiven CT-Befunde.

Material und Methoden In dieser retrospektiven monozentrischen Studie an einer Universitätsklinik mit Abteilung für Kinderradiologie wurden 2019 Thorax-CTs (973 Patienten; Altersmedian 10,5 Jahre; Spannweite: 2 Tage bis 17,9 Jahre) hinsichtlich klinischer Daten analysiert, einschließlich Zuweiserinformationen, primärer Fragestellungen oder Verdachtsdiagnosen und CT-Befunde. Es wurde festgehalten, ob die klinische Fragestellung beantwortet wurde, ob die Verdachtsdiagnose bestätigt oder ausgeschlossen wurde und ob Nebenbefunde (klinisch signifikant oder irrelevant) festgestellt wurden.

Ergebnisse Die größte klinische Untergruppe bestand aus hämatoonkologischen Fällen ( $n=987$ ) mit häufiger Fragestellung eines pulmonalen Infektfokus (66\% in dieser Gruppe). Insgesamt lieferte die CT in 97,6\% aller CTs konklusive Resul- tate. In 1380 CTs (70\%) wurde die Verdachtsdiagnose bestätigt. In 406/2019 Fällen (20\%) wurde weder ein primärer Befund noch ein Nebenbefund festgestellt. In 8 von 9 klinischen Kategorien war der Anteil der positiven Ergebnisse über $50 \%$. Vorwiegend negative Ergebnisse (110/179; 61\%) traten bei Patienten vor Stammzelltransplantation auf. In der Gruppe der Traumapatienten lieferten 81/144 der Untersuchungen (57\%) positive Ergebnisse, einschließlich mehrerer kombinierter Verletzungen $(n=23)$. 222/396 aller Nebenbefunde (56\%) wurden als klinisch relevant eingestuft.

Schlussfolgerung Die pädiatrische Thorax-CT in spezialisierten Zentren hat eine hohe diagnostische Aussagekraft im Hinblick auf konklusive Untersuchungsergebnisse. Zur Verbesserung der Effizienz der Methode und Prävention unnötiger CTUntersuchungen ist die klinische Beurteilung prä-CT entscheidend.

\section{Kernaussagen:}

- Die pädiatrische Thorax-CT in spezialisierten Zentren hat eine hohe diagnostische Aussagekraft.

- Die CT kann in klinisch komplexen Situationen relevante Veränderungen neben der Arbeitshypothese erkennen.

- Die klinische Beurteilung prä-CT ist unentbehrlich, insbesondere bei Verdacht auf eine Pneumonie.

\section{ABSTRACT}

Purpose To estimate the effectiveness and efficiency of chest $\mathrm{CT}$ in children based on the suspected diagnosis in relation to the number of positive, negative, and inconclusive $\mathrm{CT}$ results. Materials and Methods In this monocentric retrospective study at a university hospital with a division of pediatric radiology, 2019 chest CT examinations (973 patients; median age: 10.5 years; range: 2 days to 17.9 years) were analyzed with regards to clinical data, including the referring department, primary questions or suspected diagnosis, and CT findings. It was identified if the clinical question was answered, whether the suspected diagnosis was confirmed or ruled out, and if additional findings (clinically significant or minor) were detected.

Results The largest clinical subgroup was the hematooncological subgroup ( $n=987)$, with frequent questions for inflammation/pneumonia (66\% in this subgroup). Overall, CT provided conclusive results in $97.6 \%$ of all scans. In 1380 scans (70\%), the suspected diagnosis was confirmed. In 406/2019 
cases (20\%), the CT scan was negative also in terms of an additional finding. In 8 of 9 clinical categories, the proportion of positive results was over $50 \%$. There were predominantly negative results $(110 / 179 ; 61 \%)$ in pre-stem cell transplant evaluation. In the subgroup of trauma management, 81/ 144 exams ( $57 \%$ ) showed positive results, including combined injuries ( $n=23) .222 / 396(56 \%)$ of all additional findings were estimated to be clinically significant.

Conclusion In a specialized center, the effectiveness of pediatric chest CT was excellent when counting the conclusive results. However, to improve efficiency, the clinical evaluation before imaging appears crucial to prevent unnecessary CT examinations.

\section{Key Points:}

- Pediatric chest CT in specialized centers has a high diagnostic value.

- CT identifies relevant changes besides the working hypothesis in clinically complex situations.

- Pre-CT clinical evaluation is crucial, especially in the context of suspected pneumonia.

\section{Citation Format}

- Esser M, Tsiflikas I, Kraus MS et al. Effectiveness of Chest CT in Children: CT Findings in Relation to the Clinical Question. Fortschr Röntgenstr 2022; 194: 281-290

$\begin{array}{ll}\text { ABBREVIATIONS } \\ \text { CF } & \text { Cystic Fibrosis } \\ \mathrm{CI} & \text { Confidence Interval } \\ \mathrm{CT} & \text { Computed Tomography } \\ \text { ECG } & \text { Electrocardiography } \\ \text { HSCT } & \text { Hematopoietic Stem Cell Transplantation } \\ \text { MRI } & \text { Magnetic Resonance Imaging }\end{array}$

\section{Introduction}

In the last decades, computed tomography (CT) has emerged as a basic diagnostic tool in the evaluation of thoracic disease in many clinical subgroups of children. It plays a significant role in the characterization of various pulmonary and mediastinal pathologies and pleural and chest wall anomalies [1-3]. Therefore, there is no doubt about the efficacy of pediatric chest CT [3, 4]. However, especially after the introduction of modern and fast CT scanners, there has been an increased number of CT scans in pediatric patients [5].

Although the role of chest $\mathrm{CT}$ in childhood has been addressed in diagnostic guidelines and regulations for different diseases, several studies have suggested overuse of CT [6-8]. Hence, the efficiency in terms of radiation exposure is questionable. Some studies concentrated on trends in pediatric emergency departments, including patients with blunt chest trauma and brain CT $[8,9]$. Various reasons for unnecessary CT scans have been reported with the redundant repetition of scans or the preference of CT over other imaging modalities with comparable diagnostic performance (e. g., ultrasound and MRI) $[8,10]$. In solid tumors or in chronic lung disease (e.g., cystic fibrosis), some reports have recommended omitting standard surveillance by chest $\mathrm{CT}$ in asymptomatic patients or at least reducing routine follow-up imaging in cases in which the clinical impact is limited [11-13]. Thus, to justify $\mathrm{CT}$, referrers have to provide adequate medical information relevant to the requested exposure. A detailed patient history allows a deeper understanding of symptoms, and lab results may, for example, guide towards an infectious disease. Also, the results of prior diagnostic procedures should be considered. Facing the wide range of thoracic pathologies in children and the expanding diagnostic capabilities of chest CT, careful patient preselection seems mandatory to avoid unnecessary examinations and maintain the adequacy of the technique in the respective clinical scenario [14]. In this context, the interdependence between justifying CT by clinical question and image interpretation can be understood as a critical factor for the clinical effectiveness of chest CT.

As an essential factor, numerous studies have been published dealing with technical aspects, radiation exposure, or specific chest pathologies in children $[3,15,16]$. However, no study has analyzed the effectiveness of pediatric chest CT considering the relevance of findings with respect to the referring question to the best of our knowledge. To better understand the utility of chest CT in our institution, the purpose of this study was to estimate the effectiveness and efficiency of chest $\mathrm{CT}$ in children based on the suspected diagnosis in relation to the number of positive, negative, and inconclusive $\mathrm{CT}$ results.

\section{Materials and Methods}

This retrospective study was approved by the local ethics committee (project number 586/2019BO2), which waived the requirement for written informed consent. All study procedures were conducted under the ethical standards as laid down in the 1964 Declaration of Helsinki and its later amendments or comparable ethical standards.

\section{Patient selection}

A retrospective patient cohort was selected from our radiology information system between January 2007 and December 2014. The inclusion criteria included age under 18 years and at least one CT examination, including the chest. In all cases, the justification was authorized by a radiologist. The following patient-related data were collected for later analysis: patient age, sex, use of sedation or anesthesia, information about the referring department including the subdivision, admission to policlinic or hospital ward, and outpatient or inpatient care (see also Analysis of CT findings). 


\section{CT examination}

All CT examinations were performed at a single center with a board-certified division of pediatric radiology. During the observation time of seven years, six different $C T$ devices made by the manufacturer Siemens (Siemens Healthineers AG, Forchheim, Germany) were used. All acquisitions were performed with specific pediatric $\mathrm{CT}$ protocols determined from the division of pediatric radiology. Scan parameters were adapted to patient body weight. The CT settings followed the current standard operating procedures at the time of the examination.

Patients younger than six years were examined breathing freely, whereas children older than six years received a breathing command if they were able to follow the command. A support cushion was used in newborns and infants to ensure a stable position on the moving $\mathrm{CT}$ table. Board-certified pediatric radiologists carried out diagnostic reading. For interpretation, further clinical information was accessible for the radiologist via the in-house database, including lab results and information about the current treatment.

When intravenous contrast agent was applied, lomeprol (Cameron $400^{\circledR}$ ) or lopromid (Ultravist $370^{\circledR}$; both Bayer Healthcare Deutschland, Leverkusen, Germany) was used. The injected volume was adjusted to the patient's body weight. The injection was performed either by a dual-head power injection device (Medtronic, Saarbruecken, Germany) or via a manual injection when the amount of contrast agent was under $20 \mathrm{ml}$ (body weight $<10 \mathrm{~kg})$.

\section{Analysis of $\mathrm{CT}$ findings}

We analyzed $\mathrm{CT}$ reports concerning technical features, including the application of contrast agents. Clinical information was delivered with the request for an examination justifying the indication for CT scan. Each request for a CT examination included a primary question formulating the objective of the scan or the primary suspected diagnosis based on the symptoms, blood parameters, and preceding diagnostics (patient history). Clinical subgroups were formed according to the referring question. - Table 1 presents the inclusion criteria and settings for grouping in useful clinical categories.

The examination reports were stored electronically in the in-house database using SAP NetWeaver (SAP, Walldorf, Germany). For each examination, we identified whether the report included a useful result concerning the primary question (conclusive result) - which may also contain the exclusion of a suspected pathology - or if no statement was possible regarding the question. The latter means that neither a confirmation nor an exclusion of the suspected pathology was possible (inconclusive result). In the case of an inconclusive result, the reason for the limitation was taken from the report. Subsequently, we figured out if the report provided a positive answer to the primary clinical question - defined as a confirmation of the suspected diagnosis - or a negative answer - defined as a rejection of the suspected diagnosis. - Table 1 presents examples for positive and negative answers for each subgroup. As target values, a proportion of more than $50 \%$ positive results for each subgroup and an overall proportion of inconclusive results of fewer than $5 \%$ were estimated.
Finally, we recorded whether additional findings were detected. We classified additional findings as clinically important (major) or as a minor additional finding without clinical relevance. Examples of clinically significant findings are pneumonia, previously unknown tumors and pulmonary nodules, catheter-associated complications, like thrombosis or an abscess, and other findings which required further workup. Other additional findings were estimated as not relevant for the further clinical course of the patient, for example, preexisting rib fractures and other known osseous pathologies (e.g., spondylolysis, synostosis), or small pleural effusions and soft tissue emphysema after an intervention.

\section{Statistical Analysis}

Data were entered into Excel 2010 for Windows (Version 14.0; Microsoft Corporation, Redmond, WA). For statistical analysis, SAS jmp (version 11.1.1 for Windows, SAS Institute Inc., Cary, NC, USA) was used. The analysis involved descriptive statistics. Continuous variable data were presented as means \pm standard deviations. Data that did not follow a normal distribution were presented as median with a $95 \%$ confidence interval $(\mathrm{Cl})$. The normality Shapiro-Wilk test was applied to study the distribution of patient data. The Mann-Whitney $U$ test was used to analyze the difference in age between male and female patients. The Kruskal-Wallis $\mathrm{H}$ test was applied to study the correlation between inconclusive $C T$ results and scanner types and between inconclusive results and the use of sedation. A p-value less than 0.05 was considered to indicate statistical significance.

\section{Results}

\section{Study population}

The final patient cohort consisted of 973 patients (mean age \pm standard deviation, $10 \pm 5.6$ years; median and $95 \%$ confidence level, 10.5 years $[9.7 ; 10.2])$ including 581 male patients (10.2 years $\pm 5.6 ; 10.7$ years, $[9.9 ; 10.6])$ and 392 female patients (9.5 years $\pm 5.7 ; 10.4$ years, $[9.1 ; 9.9]$; difference between male and female, $p=0.003)$. Finally, 2019 chest CT scans were included.

\section{Technical results}

$62 \%(1251 / 2019)$ of scans were non-enhanced. Intravenous contrast agents were used in 768 CT examinations. The most prominent clinical subgroups of contrast-enhanced scans were cardiovascular $(n=140)$ and trauma patients $(n=136) .65 / 2019$ CT scans $(3.2 \%)$ were performed with sedation or anesthesia, most of them in the age group under one year $(n=37)$.

\section{Clinical information}

1457/2019 exams (72\%) were referred from in-house, which means that they were also accommodated for inpatient treatment. In 562 cases, a polyclinic requested the chest CT examination, which means that the scan was performed during an outpatient visit. 


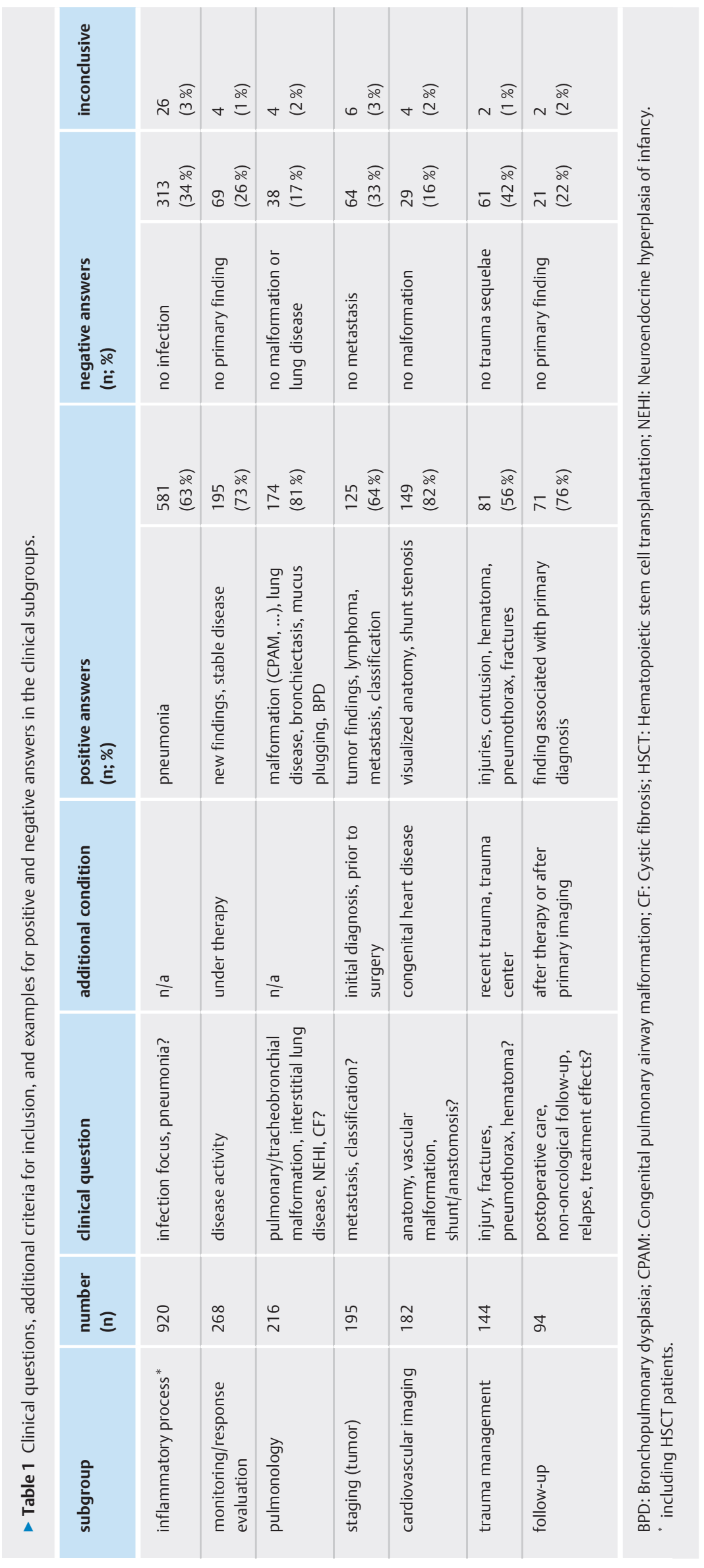




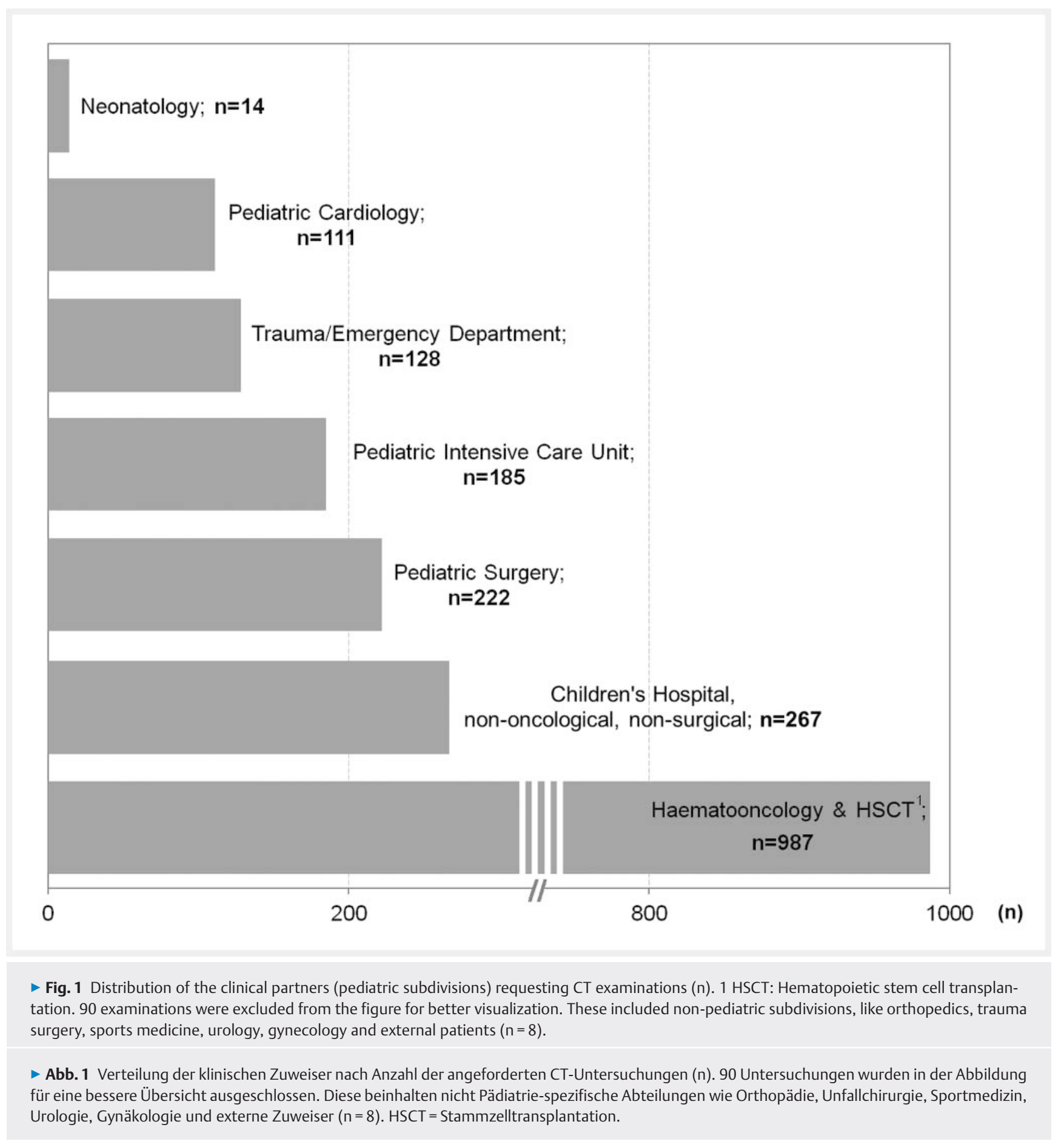

Most examinations ( $n=987$ ) were requested from the Department of Hematooncology, including the polyclinic for hematopoietic stem cell transplantation (HSCT) patients. > Fig. 1 illustrates the distribution of the subdivisions requesting the CT examinations. The subgroup of 267 non-oncological and non-surgical cases included pediatric pulmonology patients and other pathologies like pulmonary embolism and pneumothorax. There were only 36 cases with CF in this subgroup.

- Table 1 summarizes the clinical questions for the CT scans and the number of patients in each subgroup. In the subgroup of a suspected infection process ( $n=920), 361$ of the cases ( $18 \%$ of all scans) were performed to diagnose pneumonia after HSCT. Furthermore, 179 examinations ( $9 \%$ ) were carried out to exclude a pulmonary infection before HSCT.

\section{Conclusive/inconclusive results}

The proration of conclusive vs. inconclusive interpretation is listed in > Table 1. In $97.6 \%(1971 / 2019)$ of the CT scans, the primary question was answered sufficiently, or the suspected diagnosis was ruled out. Only in 48 examinations (2.4\%), the interpretation 


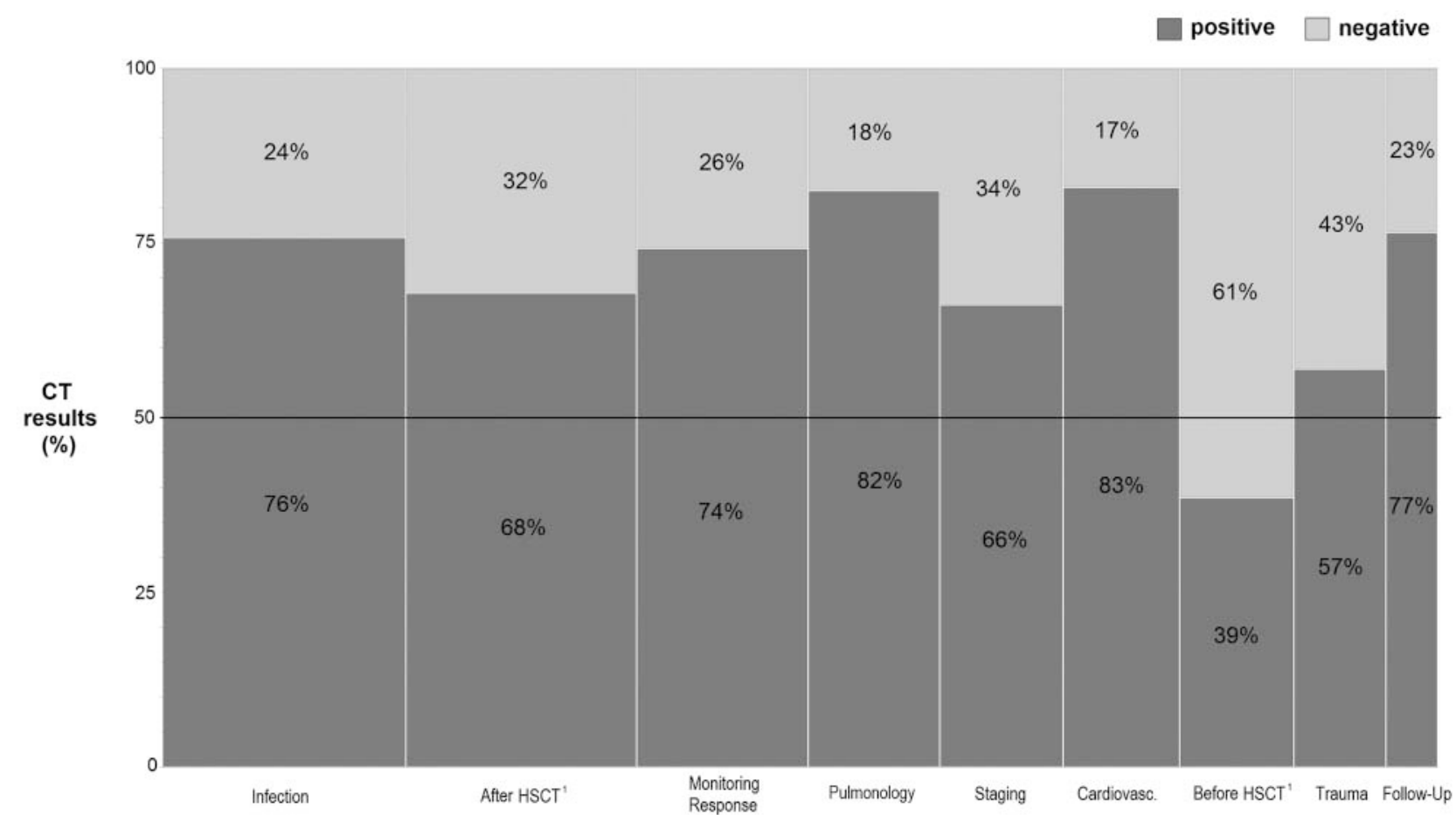

Clinical Subgroup

- Fig. 2 Proportion of positive and negative results of the CT examinations in the different clinical subgroups. The width of the boxes corresponds to the number of patients in the different clinical subgroups. The $50 \%$ line is highlighted to emphasize differences between the categories. Inconclusive results were excluded from this figure. $1 \mathrm{HSCT}$ : Hematopoietic stem cell transplantation.

- Abb.2 Anteil positiver und negativer Befunde der CT-Untersuchungen in den verschiedenen klinischen Untergruppen. Die Breite der Kästen richtet sich nach der Anzahl der Patienten in der jeweiligen Untergruppe. Die $50 \%$-Linie ist dargestellt, um die Unterschiede zwischen den Untergruppen zu verdeutlichen. Inkonklusive Ergebnisse wurden für diese Darstellung ausgeschlossen. HSCT = Stammzelltransplantation.

was inconclusive. Typical examples of inconclusive results were impaired vascular contrast or breathing artifacts so that the area of interest (a pulmonary nodule or cardiac anatomy) was not assessable. In one case, CT pointed towards fungal pneumonia, while bronchoalveolar lavage remained negative. There was no correlation between the use of different scanner types and the number of inconclusive results $(p=0.20)$. One inconclusive $C T$ in the subgroup of patients with sedation $(1 / 66 ; 1.5 \% ; p=0.65)$ resulted from an unclear cystoid mass next to the esophagus without a visible connection to the esophageal lumen proving to be an esophageal duplication cyst in endoscopy (18-month-old girl).

\section{CT findings in the subgroups}

The reports in 595 examinations (30\%) rejected the suspected diagnosis. - Table 1 shows the number of positive and negative answers with clinical examples for each subgroup. In 8 of 9 categories the proportion of positive interpretations was larger than $50 \%$ ( Fig. 2). Three categories containing infection and pneumonia, lung imaging after HSCT, and response assessment showed a proportion of positive results of $68 \%$ to $76 \%$. Only in the category "prior to HSCT", $61 \%$ of the examinations in this group had a negative result and confirmed the suspected diagnosis "normal lung."

In the category "trauma management", most of the thoracic findings were combined thoracic injuries with more than one thoracic pathology related to chest trauma $(n=23)$. The most frequent combination was lung contusion with pneumothorax and rib fractures $(n=12)$. Other complex injuries included mediastinal hematoma $(n=4)$ and diaphragmatic rupture $(n=2)$. Single findings referred to spinal fractures $(n=13)$, mediastinal hematoma $(n=3)$, hemothorax $(n=2)$, and pulmonary laceration $(n=2)$. Further findings included single lung contusions $(n=12)$, rib fractures $(n=7)$, and clavicle fractures $(n=6)$ without other pathologies.

\section{Additional findings}

In 396 scans ( $20 \%$ of all scans), an additional finding was detected. 222 of the additional findings (56\%) were classified as clinically important. For example, non-enhanced CT with the question for pulmonary metastasis in an immunocompromised patient showed consolidations with peripheral ground-glass attenuation and additional centrilobular nodules indicating fungal infection 


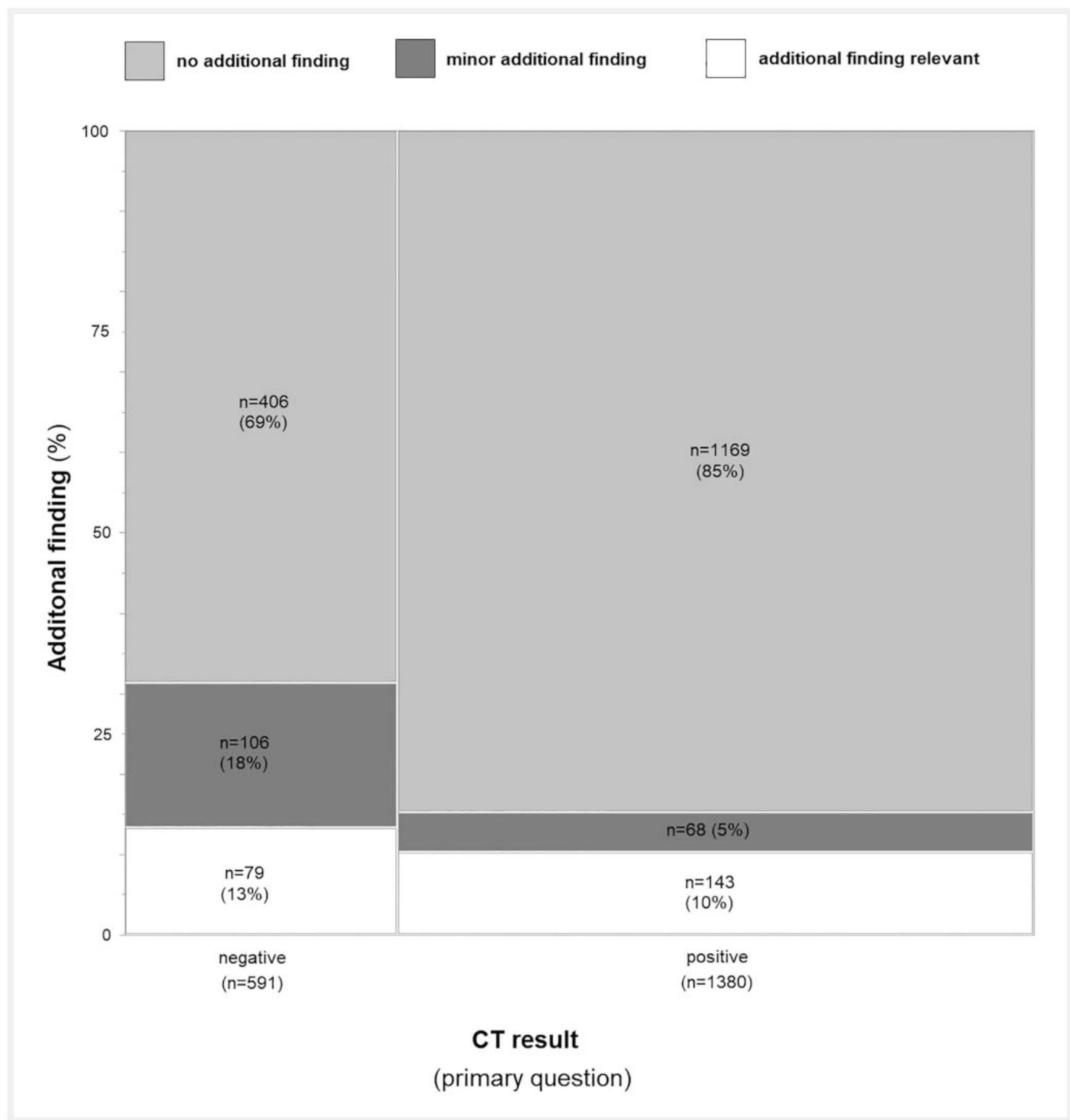

- Fig. 3 Correlation of the additional findings to the CT results regarding the primary question (positive or negative).

- Abb. 3 Korrelation zwischen den Nebenbefunden und den CT-Befunden mit Bezug zur primären klinischen Fragestellung (positiv oder negativ).

such as aspergillosis. Afterwards, oral antimycotic therapy was started.

The remaining 174 findings (44\%) were classified as minor additional findings. As an example, a small pleural fluid collection was detected after thoracoscopic wedge resection of a pulmonary metastasis as a postoperative finding.

- Fig. 3 presents a correlation of the additional findings to the $\mathrm{CT}$ results regarding the primary question. There were 79 exami- nations with a negative result concerning the primary referring reason, but with a relevant additional finding. 24/79 in this subgroup were examined for staging or tumor response assessment. In 406/2019 (20\%) examinations, neither a primary nor an additional finding was reported, which means that thoracic morphology was normal. In the subgroup of scans with a negative result and a minor or no additional finding $(n=512), 204$ examinations 
were performed before or after an HSCT. 60 scans in this subgroup were carried out during trauma management.

\section{Discussion}

This study provides the most extensive analysis of chest CT reports in children concerning clinical data and CT findings. In general, large-scale prospective studies to identify influences of imaging on treatment regimens are challenging to establish in a pediatric patient cohort due to ethical reasons (individual benefit vs. risks of radiation exposure). The cohort's heterogeneity was accepted to illuminate the full spectrum of chest CT findings in a wide range of patients.

As expected, our analysis yielded useful results proving that chest CT in children is a powerful imaging tool when considering the clinical situation and possible relevance for therapy. Overall, $98 \%$ of all chest CT examinations in our study provided conclusive interpretation by either confirming or excluding the suspected pathology. Thus, our presumed target value of $<5 \%$ inclusive reports was achieved, supporting the effectiveness of the method in our daily routine. As the pre-CT suspected diagnosis was predominantly (>50\%) confirmed in nearly all of the clinical categories, we assumed that the primary indications for CT were adequately chosen ( $\triangleright$ Fig. 2 ).

Most scans in our study were performed without a contrast agent (62\%). In line with this, the most frequent indication for chest CT was the imaging of suspected pulmonary inflammation (e. g., pneumonia; $46 \%$ ). In these subgroups, including examinations in the context of HSCT, $63 \%$ of the scans proved positive for an inflammatory process ( $\triangleright$ Table 1 ). Since chest CT may also limit the differential diagnosis or suggest the underlying pathology, e. g., a pattern suggesting viral pneumonia or identifying specific morphological features of tuberculosis, it can be assumed that the positive results may encourage further examinations or bronchoalveolar lavage or even enable optimization of an anti-infective therapy $[17,18]$. Regarding the high proportion of this category in our cohort, it should be kept in mind that CT should be used to distinguish between pneumonia and other pathologies, like pulmonary hemorrhage or congenital anomalies. Besides, as immunocompromised children suffer from pneumonia more frequently and the rate of severe complications is high, the threshold for performing chest CT should be lower compared to immunocompetent children [19]. In this context, our results point towards an interesting subgroup of 179 CT examinations ( $9 \%$ of all scans) before an HSCT to exclude a pulmonary infection. Children undergoing HSCT are at increased risk of opportunistic infections, e.g., invasive fungal infections, due to prolonged immunosuppression [20]. Nevertheless, the practice of chest CT screening to reduce the risk of post-HSCT infections is not universal. Only one study from the St. Jude Children's Research Hospital evaluated the clinical utility of pre-HSCT chest CT screening [21]. Authors stated that the indication of pre-transplantation evaluation by chest CT should consider the individual patient's history, previous treatments, and the present clinical risk for a pulmonary infection to avoid unnecessary medical evaluations or a delay of transplant [21]. As our Department for Oncology follows the St. Jude Children's Research Hospital's experiences for referring to CT before HSCT, we had a relatively large group of patients in this investigation. Predominantly (61\%) in this subgroup, CT ruled out any evidence of a silent inflammatory disease. This is in line with the results of the study of Kasow et al. with normal chest CT in $56 \%$ of the allo-recipients $(n=62)$. However, the proportion of positive scans in our study (39\%) is rather high since unrecognized infection may be fatal for these patients. Therefore, in this subgroup, CT may be justified even more than in a non-HSCT setting. Although not surprisingly, more positive results occurred after HSCT (68\% positive findings). In summary, more than $50 \%$ of the scans were found without relevant findings in the context of HSCT. Therefore, to increase the efficiency of CT in this group, we support the recommendation of Kasow et al. to carefully evaluate the indication for CT if the patient is asymptomatic and to consider co-factors like medication and the present risk for a pulmonary infection [21].

Apart from the established application such as in congenital anomalies, malignant diseases, or interstitial lung disorders, chest $\mathrm{CT}$ is the imaging reference standard also in patients with cystic fibrosis [22, 23]. However, in this patient group, pulmonary MRI has now been accepted as a radiation-free modality offering anatomical and functional details with impacts on therapy management [24-27]. This may also explain the relatively low number of CF cases in our study $(n=36)$. Rising opportunities in chest imaging require us, even more than before, to choose the suitable imaging modality according to the clinical context and to consider each patient's radiation history when indicating repeated chest imaging [28]. In the context of improved outcomes and increasing life expectancy - not only concerning CF, but also in pediatric hematooncology (e. g., lymphoma) - imaging surveillance carried out for a longer period over a lifetime should be assessed critically $[12,13]$. The use of CT in an inappropriate clinical situation may result in false-positive results (pseudo-disease) or incidentalomas that are not relevant for treatment or may even hamper an immediate target-oriented therapy [8]. Risk-adapted strategies for imaging surveillance have been proposed for survivors of childhood lymphoma [11].

Concerning the prevention of unnecessary CT in children, former reports have dealt with the clinical benefit of chest CT in children with blunt trauma [29]. A chest radiograph may not be the superior imaging modality but may be sufficient to answer the clinical question and exclude relevant pathologies in a trauma setting. Therefore, $\mathrm{CT}$ in pediatric chest trauma patients is frequently used as a confirmatory diagnostic tool [9]. This applies to our study only to a limited extent. Positive results were found in $57 \%$ of the subgroup of trauma patients ( $\triangleright$ Fig. 2 ). As most of the findings described combined thoracic pathologies or other complex injuries, like spinal fractures or diaphragmatic rupture, it can be estimated that chest $\mathrm{CT}$ added clinical information in the trauma setting in most cases. The decision to perform chest CT should be based on interdisciplinary clinical evaluation including close communication between the radiologist and the clinical care team [30].

48 scans in our study delivered inconclusive results. As technical issues like reduced vascular contrast or breathing artifacts were frequent reasons for impaired assessment, these cases 
draw attention towards the necessity of individually adjusting scan protocols and optimizing examination conditions in a pediatric setting. These aspects seem especially important for the subgroup of cardiovascular patients. Concerning cardiovascular pathologies in our study, indications were often indisputable and clinical usefulness was evident in children with congenital heart disease for the visualization of anatomy (82\%) ( $\$$ Table 1 ). However, in this patient group, the scan protocols and technical details, like the use of ECG or high-pitch scanning, are of particular concern to avoid a reduction of image quality [31].

Our study demonstrated a small number of CT examinations $(n=79)$ which rejected the primary suspected diagnosis, but an additional finding with clinical significance was detected ( $\triangleright$ Fig.3). This means that chest CT may also identify relevant changes besides the reason for the referral. Also, in cases with a low pretest probability for disorders, the findings could substantially impact clinical decisions. However, this does not justify random use of CT but can be helpful in complex situations. $30 \%$ of the patients in this subgroup $(n=24)$ were examined for staging or tumor response assessment in malignant disorders in which complications (tumor-associated or therapy-related) are more likely to occur.

Our study has several limitations that require discussion. The most critical limitation is that our study was set up at a single center. Consequently, it depends on the local standard of care, limiting the generalization of the study findings. Undoubtedly, the classification of examinations into different clinical categories somehow simplifies many real-life situations in clinically complex cases with more than one question to be answered by the CT examination or different suspected diagnoses. Nevertheless, the statistical relevance of simplification may be expected to be low in a study population of more than 2000 patients. The CT findings were not compared to other imaging modalities.

In summary, our study confirms that pediatric chest CT in specialized centers has a high effectiveness when the application is based on sufficient clinical data and well-known patient history. The pre-CT evaluation appears crucial for preventing unnecessary CT examinations, especially in the context of suspected pneumonia or trauma management. Thus, the risk/benefit ratio (efficacy) should be looked at even more closely. Furthermore, other imaging modalities, mainly MRI, should be considered if the individual clinical situation is suitable.

\section{CLINICAL RELEVANCE:}

- Chest CT in children has high clinical value, also in clinically complex situations.

- Knowledge of clinical parameters and patient history is essential for the indication and evaluation of pediatric chest CT.

- In an HSCT setting, CT indication and interpretation should consider the exact transplantation phase.

\section{Conflict of Interest}

The authors declare that they have no conflict of interest.

\section{References}

[1] Donnelly LF, Lucaya J, Strife JL. Pediatric chest imaging. Chest imaging in infants and children. In: CT of acute pulmonary disease: infection, infarction, and trauma. Berlin, Heidelberg: Springer; 2008: 145-164

[2] Paterson A. Imaging evaluation of congenital lung abnormalities in infants and children. Radiol Clin North Am 2005; 43: 303-323

[3] Lobo L, Antunes D. Chest CT in infants and children. Eur J Radiol 2013; 82: 1108-1117

[4] Haynes B. Can it work? Does it work? Is it worth it? The testing of healthcare interventions is evolving. BMJ 1999; 319: 652-653

[5] Brenner DJ, Hall EJ. Computed tomography-an increasing source of radiation exposure. N Engl J Med 2007; 357: 2277-2284

[6] Shahi V, Brinjikji W, Cloft H] et al. Trends in CT Utilization for Pediatric Fall Patients in US Emergency Departments. Acad Radiol 2015; 22: 898-903

[7] Eren B, Karagoz Guzey F. Is spinal computed tomography necessary in pediatric trauma patients? Pediatr Int 2020; 62: 29-35

[8] Ohana O, Soffer S, Zimlichman E et al. Overuse of CT and MRI in paediatric emergency departments. Br J Radiol 2018; 91: 20170434

[9] Holl EM, Marek AP, Nygaard RM et al. Use of Chest Computed Tomography for Blunt Pediatric Chest Trauma: Does It Change Clinical Course? Pediatr Emerg Care 2020; 36: 81-86

[10] Klang E, Beytelman A, Greenberg D et al. Overuse of Head CT Examinations for the Investigation of Minor Head Trauma: Analysis of Contributing Factors. Am Coll Radiol 2017; 14: 171-176

[11] Voss SD, Cairo MS. Surveillance imaging in pediatric lymphoma. Pediatr Radiol 2019; 49: 1565-1573

[12] Gilchrist FJ, Buka R, Jones M et al. Clinical indications and scanning protocols for chest CT in children with cystic fibrosis: a survey of UK tertiary centres. BMJ Paediatr Open 2018; 2: e000367

[13] Federico SM, Brady SL, Pappo A et al. The role of chest computed tomography $(\mathrm{CT})$ as a surveillance tool in children with high-risk neuroblastoma. Pediatr Blood Cancer 2015; 62: 976-981

[14] Howe J, Fitzpatrick CM, Lakam DR et al. Routine repeat brain computed tomography in all children with mild traumatic brain injury may result in unnecessary radiation exposure. J Trauma Acute Care Surg 2014; 76: 292-295; discussion 295-296

[15] Esser M, Hess S, Teufel M et al. Radiation Dose Optimization in Pediatric Chest CT: Major Indicators of Dose Exposure in 1695 CT Scans over Seven Years. Rofo 2018; 190: 1131-1140

[16] Martine RJ, Santangelo T, Colas L et al. Radiation dose levels in pediatric chest CT: experience in 499 children evaluated with dual-source singleenergy CT. Pediatr Radiol 2017; 47: 161-168

[17] Kunihiro Y, Tanaka N, Kawano R et al. Differential diagnosis of pulmonary infections in immunocompromised patients using high-resolution computed tomography. Eur Radiol 2019; 29: 6089-6099

[18] Lee J], Chong PY, Lin CB et al. High resolution chest CT in patients with pulmonary tuberculosis: characteristic findings before and after antituberculous therapy. Eur J Radiol 2008; 67: 100-104

[19] Andronikou S, Goussard P, Sorantin E. Computed tomography in children with community-acquired pneumonia. Pediatr Radiol 2017; 47: 1431-1440

[20] Junghanss C, Marr KA, Carter RA et al. Incidence and outcome of bacterial and fungal infections following nonmyeloablative compared with myeloablative allogeneic hematopoietic stem cell transplantation: a matched control study. Biol Blood Marrow Transplant 2002; 8: 512-520 
[21] Kasow KA, Krueger ], Srivastava DK et al. Clinical utility of computed tomography screening of chest, abdomen, and sinuses before hematopoietic stem cell transplantation: the St. Jude experience. Biol Blood Marrow Transplant 2009; 15: 490-495

[22] Tiddens HAWM, Kuo W, van Straten $M$ et al. Paediatric lung imaging: the times they are a-changin'. Eur Respir Rev 2018; 28: 27

[23] Lee ], Sanchez TR, Zhang Y et al. The role of high-resolution chest CT in the diagnosis of neuroendocrine cell hyperplasia of infancy - A rare form of pediatric interstitial lung disease. Respir Med Case Rep 2015; 16: 101103

[24] Schaefer JF, Hector A, Schmidt K et al. A semiquantitative MRI-Score can predict loss of lung function in patients with cystic fibrosis: Preliminary results. Eur Radiol 2018; 28: 74-84

[25] Fleischer S, Kraus MS, Gatidis S et al. New severity assessment in cystic fibrosis: signal intensity and lung volume compared to LCl and FEV1: preliminary results. Eur Radiol 2020; 30: 1350-1358

[26] Kraus MS, Teufel M, Esser M et al. Differing Pulmonary Structural Abnormalities Detected on Pulmonary MR Imaging in Cystic Fibrosis Patients with Varying Pancreatic Function. Rofo 2020; 192: 567-575
[27] Dournes G, Menut F, Macey J et al. Lung morphology assessment of cystic fibrosis using MRI with ultra-short echo time at submillimeter spatial resolution. Eur Radiol 2016; 26: 3811-3820

[28] Westra S]. The communication of the radiation risk from CT in relation to its clinical benefit in the era of personalized medicine: part 2: benefits versus risk of CT. Pediatr Radiol 2014; 44 (Suppl. 3): 525-533

[29] Holscher CM, Faulk LW, Moore EE et al. Chest computed tomography imaging for blunt pediatric trauma: not worth the radiation risk. J Surg Res 2013; 184: 352-357

[30] Moore MA, Wallace EC, Westra S]. Chest trauma in children: current imaging guidelines and techniques. Radiol Clin North Am 2011; 49: 949-968

[31] Esser M, Gatidis S, Teufel M et al. Contrast-Enhanced High-Pitch Computed Tomography in Pediatric Patients Without Electrocardiography Triggering and Sedation: Comparison of Cardiac Image Quality With Conventional Multidetector Computed Tomography. J Comput Assist Tomogr 2017; 41: 165-171 